\title{
Salud mental y miedo a la separación en mujeres víctimas de violencia de pareja
}

\author{
Esther Rivas-Rivero* y Enrique Bonilla-Algovia \\ Universidad de Alcalá
}

- Recibido: 02 - 09 - 2019 - Aceptado: 16 - 12 - 2019 . Avance Online: 20 - 12 - 2019

RESUMEN: El trastorno por estrés postraumático (TEPT) es un diagnóstico altamente reportado entre las mujeres víctimas de violencia de pareja cuya sintomatología podría tener graves consecuencias en los procesos de separación del agresor. En la investigación participaron 61 mujeres de León (Nicaragua), con una media de edad de 32 años, que habían sufrido maltrato de pareja, a quienes se aplicó una entrevista estructurada. El acceso a la muestra se realizó en colaboración con la Comisaría de la Mujer y otros dispositivos de apoyo a las víctimas. Los resultados reportan que las participantes presentaban un deterioro en la salud mental como consecuencia de la frecuencia del maltrato, así como la influencia del TEPT en el miedo a la ruptura con la relación. Estudiar la salud mental y el miedo a la separación del agresor es fundamental en la intervención con víctimas de violencia de pareja, pues el daño psicológico podría afectar al mantenimiento de la relación abusiva.

PALABRAS CAVE: Violencia de pareja, Trastorno por estrés postraumático, Ansiedad, Depresión, Victimización.

\section{Mental health and fear of separation in women victims of partner violence}

ABSTRACT: Posttraumatic stress disorder (PTSD) is a highly reported diagnosis among women victims of intimate partner violence whose symptoms could have serious consequences in the processes of separation from the aggressor. The investigation involved 61 women from León (Nicaragua), with an average age of 32 years, who had suffered maltreatment, to whom a structured interview was applied. Access to the sample was made in collaboration with the Women's Police Station and other support devices for victims. The results show that the women in the sample presented a deterioration in mental health as a result of the frequency of abuse, as well as the influence of PTSD in fear of rupture with the relationship. Studying mental health and fear of separation from the aggressor is essential in the intervention with victims of intimate partner violence, as psychological damage could affect the maintenance of the abusive relation.

KEY WORDS: Intimate Partner violence, post-traumatic stress disorder, Anxiety, Depression, Victimization.

La violencia contra las mujeres en la pareja es un problema de salud pública y una violación contra los derechos humanos que ocurre en la mayoría de las sociedades, culturas y niveles socioeconómicos. Esta incluye la violencia física y sexual, el acoso y el maltrato psicológico por parte de una pareja actual o anterior (Sparrow, Kwan, Howard, Fear, y MacManus, 2017). La

*Correspondence: Esther Rivas Rivero.

Universidad de Alcalá

CP: 28801, Alcalá de Henares, España.

E-mail: esther.rivas@uah.es

(c) 2020 Sociedad Universitaria de Investigación en Psicología y Salud. Publicado por Consejo General de Colegios Oficiales de Psicólogos, España. Este es un artículo Open Access
bajo la CC BY-NC-ND licencia (http://creativecommons.org/licencias/by-nc-nd/4.0/) violencia contra las mujeres en el ámbito de la pareja varía entre los distintos países, si bien, la prevalencia mundial oscila entre el $29 \%$ y el $62 \%$ (Silva y Azeredo, 2019). Además, la depresión, la ansiedad y el trastorno por estrés postraumático (TEPT) son cuadros clínicos frecuentemente reportados entre quienes la padecen (Khalifeh, Oram, Trevillion, Johnson, y Howard, 2015; Sparrow et al., 2017). Por lo tanto, este tipo de violencia podría generar problemas de salud psicológica que interferirían de forma negativa en la vida cotidiana de las mujeres que la sufren (Echeburúa, Amor, Sarasua, Zubizarreta, Holgado-Tello, y Muñoz, 2016). 
Concretamente, el TEPT es uno de los diagnósticos más frecuentes en la violencia contra las mujeres, con tasas que oscilan entre el 16\% al 57\% (Golding, 1999; Greene, Chan, McCarthy, Wakschlag, y Briggs-Gowan, 2018; Mertin y Mohr, 2000). Para otros, su prevalencia puede alcanzar el 80\% (Riggs, Caulfield, y Street, 2000). Este trastorno deriva en recuerdos y sueños angustiosos recurrentes respecto al suceso, malestar psicológico intenso ante la exposición a estímulos asociados al acontecimiento traumático, así como reacciones fisiológicas intensas al recordarlo (APA, 2014). Puede estar condicionado por la gravedad y la frecuencia de dicha violencia (Mbalo, Zhang, y Sam, 2017), la mayor o menor vulnerabilidad de la víctima ante el acontecimiento traumático, la concomitancia con otros problemas, el historial de victimización y la falta de apoyo social ante tales circunstancias (Echeburúa et al., 2016). Es importante señalar que este diagnóstico requiere de una cuidadosa evaluación, ya que es comórbido con depresión, ansiedad y otros trastornos, y en la evaluación forense a menudo es problemático por las limitaciones de los instrumentos y la aplicación de una evaluación clínica tradicional no válida en determinados contextos (Arce, Fariña, y Vilariño, 2015; Fariña, Arce, Vilariño, y Novo, 2014).

Algunos estudios han encontrado que quienes desarrollan el TEPT tienen poco control de la situación y reportan un deterioro significativo en el funcionamiento, aun cuando no cumplen con la totalidad de los criterios diagnósticos (Sullivan, Weiss, Price, Pugh, y Hansen, 2018). Además, las conductas amenazantes y el miedo que se instaura en las mujeres ante el temor a más violencia están altamente relacionados con el TEPT (Sullivan y Weiss, 2017). De este modo, el miedo parece tener un papel central y podría influir en los procesos de separación (Sarasúa, Zubizarreta, Echeburúa, y Corral, 2007). Goodman, Bennet y Dutton (1999) afirman que el miedo se asocia al mantenimiento de la relación, pues este inhibe la ruptura del vínculo con el agresor entre quienes lo desarrollan (Ruiz, López, Hernández, Castañeda, y Aguila, 2013).

Cuando las mujeres víctimas de violencia en el seno de la pareja deciden terminar con la relación, entran en el momento de mayor peligrosidad (Heise, Ellsberg, y Gotemoeller, 1999). Algunas investigaciones refieren que es ante situaciones extremas, como la amenaza de muerte, cuando las mujeres víctimas inician procesos de separación (Ruiz et al., 2013). Por ello, la ruptura con la relación de violencia es uno de los momentos más difíciles para las víctimas, especialmente durante los seis meses posteriores a la toma de esta decisión (Lerner y Kennedy, 2000), de ahí que algunos autores hayan denominado a este periodo "ruta crítica" (Sagot y Carcedo, 2000). Además, la relevancia que tiene la ruptura hace que, más que como un hecho puntual, se tenga que concebir como un proceso (Anderson y Saunders, 2003).

Aun con todo, muchas mujeres consiguen perder el miedo y poner fin a la relación cuando cuentan con apoyo social y con profesionales que las escuchan y orientan (Bosch, Ferrer, Ferreiro, y Navarro, 2013; Rivas, Panadero, Bonilla, Vázquez, y Vázquez, 2018), aunque hay pocas investigaciones que aborden la relación entre la salud psicológica como consecuencia del maltrato y el miedo a la separación. Analizar estas variables es importante, puesto que podrían influir significativamente en el funcionamiento en diversos contextos (social, familiar y psicológico) (Luna, Rodríguez, y Hernández, 2018). Hay que añadir que, a la salud psicológica se añaden múltiples variables que se vinculan al mantenimiento de la mujer en la relación, siendo las más reportadas la dependencia económica y/o afectiva de la víctima hacia el agresor, los sentimientos de culpa, la falta de conciencia de estar siendo víctima de maltrato y la presión social respecto a mantener a la familia unida (Bosch, Ferrer, Alzamora, y Navarro, 2005; Watts y Zimmerman, 2002).

Algunos estudios con víctimas de violencia de pareja de Centroamérica revelan que el miedo inhibe la separación (Bolaños et al., 2009; Ruiz et al., 2013), no obstante, este tendría un origen multicausal que podría estar relacionado con una amplia variedad de factores, habiéndose demostrado que el apoyo procedente de la familia influye positivamente en la ruptura con la relación de maltrato (Rivas 
et al., 2018). Hay que señalar que son pocos los estudios en los que se analice el impacto de la violencia en mujeres procedentes de países de bajos ingresos (Ellsberg y Emmelin, 2014), de modo que esta realidad está pendiente de ser visibilizada en más investigaciones. Además, los resultados que se reportan en regiones con niveles altos de ingresos no reflejan la realidad de otras sociedades en las que no existen respuestas del sector de la justicia, ni prestación de servicios de apoyo integral en la eliminación de la violencia contra las mujeres.

En Nicaragua, puesto 124 en el índice de desarrollo humano (PNUD, 2016), en torno al $52 \%$ de las mujeres ha experimentado violencia física o sexual alguna vez en su vida (Boyce, Zeledón, Téllez, y Barrington, 2016). La investigación realizada por Salazar, Valladares, Öhman y Högberg (2009), con mujeres nicaragüenses víctimas de violencia de pareja, evidencia que los recursos sociales del entorno, el nivel educativo, la menor aceptación de los roles tradicionales de género, la baja tolerancia de la comunidad hacia la violencia contra las mujeres y la formación para identificar la violencia de pareja son mecanismos de protección en los procesos de ruptura con el agresor que aumentan el bienestar psicológico y emocional. No obstante, la revictimización de las mujeres nicaragüenses constituye un serio problema teniendo en cuenta que, en los dispositivos de atención, el personal carece de capacitación (Colombini, Mayhwe, y Watts, 2008), y a la elevada prevalencia del problema se añade la escasez de recursos de apoyo a las mujeres (Rivas, Bonilla, y Vázquez, 2019).

El objetivo del presente estudio es analizar el estado de salud psicológica en mujeres víctimas de violencia en contexto de pareja y su relación con una de las dificultades a las que se enfrentan para abandonar la relación abusiva, concretamente, el miedo a la separación. Estudiar las circunstancias de las mujeres víctimas en un país sobre el que no hay trabajos a este respecto es crucial en el análisis de esta problemática cuando no existen políticas ni estructuras que actúen en la eliminación de la violencia contra las mujeres.

\section{MÉTODO}

\section{-PARTICIPANTES}

En el estudio participaron 61 mujeres víctimas de violencia en el seno de la pareja de León (Nicaragua), una muestra sometida a un conjunto especialmente grave de situaciones estresantes relacionadas con la pobreza y la violencia, a quienes se accedió a través de la Comisaría de la Mujery de otras asociaciones que trabajan con la Policía Nacional Nicaragüense. Para la selección de las participantes se consideraron los siguientes criterios de inclusión muestral: ser mayor de 18 años, cohabitar con el agresor, que el último episodio de violencia se produjese el mes previo a la realización de la entrevista y encontrarse en situación de pobreza. En la Tabla 1 se muestran las principales características de las participantes.

La edad media de las participantes era de 32 años, dentro de un rango comprendido entre los 19 y los 54 años. Estas tenían un promedio de dos hijos/as, si bien el rango era de cero a siete hijos/as. En torno a la mitad estaban casadas o en unión de hecho estable. Más de una tercera parte de la muestra no tenía un trabajo remunerado. Hay que añadir que el nivel educativo de las participantes era bajo en la mayoría de los casos. En cuanto a la situación de violencia, el $56 \%$ padecía maltrato varias veces a la semana y una de cada cinco lo sufría diariamente. La totalidad de la muestra fue víctima de maltrato psicológico y físico y más del $70 \%$ fue forzada a mantener relaciones sexuales por parte de la pareja. Por último, en torno al $60 \%$ manifestó tener miedo a la separación del agresor.

\section{- INSTRUMENTOS}

Características sociodemográficas: Se recogió información respecto a la edad, el número de hijos e hijas, situación laboral, estado civil, nivel de escolaridad, etc. También se solicitó información respecto a la ocurrencia del maltrato y otras circunstancias relacionadas con la violencia padecida. El miedo a la separación fue una pregunta introducida ad hoc con respuesta dicotómica (Sí/No). 
Tabla 1

Características sociodemográficas.

\begin{tabular}{|c|c|c|c|}
\hline & $n$ & $\%$ & $M(D T)$ \\
\hline Edad & & & $31.69(8.482)$ \\
\hline No de hijos/as & & & $2.13(1.231)$ \\
\hline \multicolumn{4}{|l|}{ Estado civil } \\
\hline Soltera & 13 & $21.3 \%$ & \\
\hline Casada & 12 & $19.7 \%$ & \\
\hline Unión de hecho & 26 & $42.6 \%$ & \\
\hline Separada & 8 & $13.1 \%$ & \\
\hline Divorciada & 2 & $3.3 \%$ & \\
\hline \multicolumn{4}{|l|}{ Situación laboral } \\
\hline Parada & 2 & $3.3 \%$ & \\
\hline Ama de casa & 22 & $36.1 \%$ & \\
\hline Trabajadora ocasional & 18 & $29.5 \%$ & \\
\hline Trabajadora estable & 19 & $31.2 \%$ & \\
\hline \multicolumn{4}{|l|}{ Nivel de estudios } \\
\hline Sin estudios & 1 & $1.6 \%$ & \\
\hline Básicos & 50 & $81.9 \%$ & \\
\hline Educación media & 6 & $9.9 \%$ & \\
\hline Educación superior & 4 & $6.5 \%$ & \\
\hline Sin ingresos propios & 20 & $32.8 \%$ & \\
\hline Miedo a la separación & 36 & $59 \%$ & \\
\hline \multicolumn{4}{|l|}{ Frecuencia del maltrato } \\
\hline Algunas veces al mes & 14 & $23.7 \%$ & \\
\hline Varias veces a la semana & 33 & $55.9 \%$ & \\
\hline Diariamente & 12 & $20.3 \%$ & \\
\hline \multicolumn{4}{|l|}{ Tipo de violencia } \\
\hline Psicológica & 61 & $100 \%$ & \\
\hline Física & 61 & $100 \%$ & \\
\hline Sexual & 43 & $70.5 \%$ & \\
\hline
\end{tabular}

Escala de Trauma de Davidson (DTS, Davidson Trauma Scale) (Davidson et al., 1997), en la versión adaptada y validada al español (Bobes et al., 2000; Guerra, Martínez, Ahumada, y Díaz, 2013). La escala evalúa la frecuencia y la gravedad o afectación de las personas que han experimentado un trauma. Este instrumento consta de 17 ítems con formato de respuesta tipo Likert de 5 alternativas que evalúan cada uno de los síntomas recogidos en el DSM-IV del TEPT: el acontecimiento traumático es reexperimentado persistentemente a través de recuerdos, sueños, flashbacks, respuestas fisiológicas ( $B$, ítems 1 a 5 ); evitación persistente de estímulos asociados al trauma (C, ítems 6 a 12) y síntomas persistentes de aumento de la activación (D, ítems 13 a 17). Los 17 ítems son los mismos en la subescala de frecuencia y en la subescala de gravedad/afectación, sin embargo, el formato de respuesta es diferente: frecuencia (0, nunca; a 4, diariamente) y gravedad (0, nada; a 4, extremadamente alta). La puntuación oscila entre 0 y 68 puntos en frecuencia y entre 0 y 68 puntos en afectación. Por lo tanto, la puntuación total oscila entre 0 y 136, por lo que, a mayor puntuación, mayor es la incidencia del TEPT. Davidson et al. (1997) establecen como punto de corte 40 puntos, de modo que una puntuación superior indica una alta probabilidad de que la persona sufra TEPT. La media encontrada en el presente estudio fue de 41.86 puntos (DT $=24.837$ ). 
El Alfa de Cronbach según la frecuencia fue $\alpha$ $=$.945. Respecto a la intensidad o afectación fue $\alpha=.934$, siendo superior a los anteriores el obtenido en el sumatorio total de la escala $(\alpha=$.968).

Escala Kessler 10 (K-10, Kessler et al., 2002), en la versión adaptada al español y validada en México (Vargas, Villamil, Rodríguez, Pérez, y Cortés, 2011 1). Se trata de una escala unidimensional y está formada por diez preguntas específicas sobre el malestar psicológico referidas a la ansiedad y la depresión. El rango de respuesta es tipo Likert de cinco niveles (5, siempre; 4, casi siempre; 3 , a veces; 2 , casi nunca y 1 , nunca). La suma de las puntuaciones tiene un mínimo de 10 puntos y un máximo de 50 puntos. Los rangos del instrumento pueden interpretarse de acuerdo a cuatro niveles: (bajo (10-15), moderado (16$21)$, alto (22-29) y muy alto (30-50). El 50.8\% de las mujeres de la muestra puntuaron entre 22-29 puntos y el $36.1 \%$ por encima de 30 puntos, lo que indica altos niveles de ansiedad y depresión entre las participantes. El Alfa de Cronbach obtenido en el presente estudio ha sido $\alpha=.954$.

\section{-PROCEDIMIENTO}

El acceso a la muestra se obtuvo a través de profesionales de instituciones y asociaciones que trabajan con víctimas de violencia contra las mujeres de León (Nicaragua), entre las que destaca la Comisaría de la Mujer. Estas se pusieron en contacto con víctimas que acudieron a sus dependencias para buscar apoyo. Se explicaron los objetivos del estudio y se solicitó el consentimiento informado a las mujeres, quienes participaron de forma voluntaria. La recogida de información se realizó mediante una entrevista estructurada que tuvo una duración que oscilaba entre los 45 y los 80 minutos.

\section{-ANÁLISIS DE DATOS}

La base de datos se diseñó mediante el programa estadístico SPSS (IBM SPSS Statistics 24.0). También se utilizó ANOVA de un factor y se especificaron las diferencias intergrupales respecto a quienes padecían maltrato (1) algunas veces al mes, (2) varias veces a la semana y (3) diariamente mediante análisis Post-Hoc (Bonferroni). La comparación en las puntuaciones medias del DTS (Davidson et al., 1997) respecto a quienes afirmaron que tenían miedo a la separación se llevó a cabo a través de la prueba $t$ de Student para muestras independientes. Para considerar un resultado estadísticamente significativo se adoptó una probabilidad de cometer un error tipo I de $p<.05$. Para estudiar la relación entre la frecuencia de la violencia sufrida, el estrés postraumático, la ansiedad, la depresión y el miedo a la separación, se diseñó un modelo de ecuaciones estructurales (SEM, Structural Equation Models). Para ello se utilizó el programa estadístico AMOS (IBM AMOS 24.0). La normalidad multivariante se evaluó tomando en consideración los valores del Test de Mardia (Mardia, 1974). El análisis del ajuste global del modelo, ante los resultados de la normalidad multivariante, se llevó a cabo mediante la técnica de Máxima Verosimilitud (Maximum likelihood). Para determinar la calidad de ajuste se siguieron los siguientes indicadores: $\chi^{2} / \mathrm{gl} \leq$ 3; GFI, AGFI, CFI, NFI $\geq$.95, SRMR $\geq .08$, RMSEA $\leq .06$ (Schreiber, Nora, Stage, Barlow y King, 2006).

\section{RESULTADOS}

En la Tabla 2 se muestran las puntuaciones obtenidas en cada uno de los ítems del DTS. La frecuencia media de los indicadores de TEPT fue de 21.45 (DT = 12.987). La intensidad o afectación media de dichos indicadores de la escala fue de 20.48 (DT = 12.515).

En la Tabla 3 se observan las diferencias entre la frecuencia del maltrato (algunas veces al mes, varias veces a la semana y diariamente) en función de la escala DTS. En primer lugar, se encontraron diferencias estadísticamente significativas entre quienes padecieron maltrato algunas veces al mes, varias veces a la semana y diariamente y la frecuencia en los indicadores del DTS. También se hallaron diferencias entre los grupos en función de la intensidad - afectación. Finalmente, se reportan las diferencias del sumatorio global de la escala 
Tabla 2

Puntuaciones medias respecto a la frecuencia en la escala DTS.

\begin{tabular}{|c|c|c|c|c|c|}
\hline & & \multicolumn{2}{|c|}{ Frecuencia } & \multicolumn{2}{|c|}{ Intensidad } \\
\hline & & M & DT & M & DT \\
\hline Ítem 1 & $\begin{array}{l}\text { Ha tenido imágenes, recuerdos o pensamientos dolorosos acerca del } \\
\text { acontecimiento }\end{array}$ & 1.54 & 1.134 & 1.21 & .951 \\
\hline Ítem 2 & Ha tenido pesadillas con el acontecimiento & 1.59 & 1.131 & 1.31 & 1.025 \\
\hline Ítem 3 & $\begin{array}{l}\text { Ha sentido que el acontecimiento estaba ocurriendo de nuevo como si } \\
\text { lo estuviera reviviendo }\end{array}$ & 1.20 & 1.117 & 1.10 & .943 \\
\hline Ítem 4 & Se ha sentido incómoda o molesta al recordar el acontecimiento & 1.38 & 1.186 & 1.21 & .951 \\
\hline Ítem 5 & Ha tenido síntomas físicos cuando se acuerda del acontecimiento & 1.23 & 1.125 & 1.08 & 1.021 \\
\hline Ítem 6 & $\begin{array}{c}\text { Ha estado evitando algún pensamiento o sentimiento sobre el } \\
\text { acontecimiento }\end{array}$ & 1.46 & 1.149 & 1.21 & .933 \\
\hline Ítem 7 & $\begin{array}{l}\text { Ha estado evitando hacer cosas o estar en situaciones que le } \\
\text { recordaran }\end{array}$ & 1.56 & 1.057 & 1.41 & 1.726 \\
\hline Ítem 8 & Ha sido incapaz de recordar partes importantes del acontecimiento & 1.39 & 1.069 & 1.21 & 1.018 \\
\hline Ítem 9 & $\begin{array}{l}\text { Ha tenido dificultades para disfrutar de cosas o situaciones que antes } \\
\text { del acontecimiento usted consideraba placenteras }\end{array}$ & 1.36 & 1.111 & 1.36 & 1.081 \\
\hline Ítem 10 & Se ha sentido distante o alejada de la gente & 1.23 & .973 & 1.28 & 1.051 \\
\hline Ítem 11 & Se ha sentido incapaz de tener sentimientos de tristeza o afecto & 1.16 & .952 & 1.25 & 1.035 \\
\hline Ítem 12 & $\begin{array}{l}\text { Ha tenido dificultades para imaginar una vida larga y cumplir sus } \\
\text { objetivos }\end{array}$ & 1.13 & 1.087 & 1.16 & 1.098 \\
\hline Ítem 13 & Ha tenido dificultades para iniciar o mantener el sueño & 1.11 & .915 & 1.10 & .978 \\
\hline Ítem 14 & Ha estado irritable o ha tenido episodios de ira o rabia intensa & 1.11 & .933 & 1.05 & .902 \\
\hline Ítem 15 & Ha tenido dificultades de concentración & 1.23 & 1.007 & 1.15 & .980 \\
\hline Ítem 16 & $\begin{array}{l}\text { Se ha sentido nerviosa o con la sensación de estar demasiado alerta } \\
\text { de lo que está pasando }\end{array}$ & 1.11 & 1.066 & 1.11 & 1.018 \\
\hline Ítem 17 & Se ha sobresaltado o se ha asustado fácilmente & 1.18 & 1.057 & .98 & .957 \\
\hline
\end{tabular}

Tabla 3

Indicadores TEPT en función de la frecuencia del maltrato.

\begin{tabular}{|c|c|c|c|c|c|c|c|}
\hline DTS & $\begin{array}{c}\text { Frecuencia de la } \\
\text { violencia }\end{array}$ & $n$ & $m$ & $S_{x}$ & $\begin{array}{c}\text { Media } \\
\text { cuadrática } \\
\text { (Intergrupo) }\end{array}$ & $F$ & $p$ \\
\hline \multirow{3}{*}{ DTSfrecuencia } & Algunas veces/mes & 14 & 14.92 & 10.894 & & & \\
\hline & Varias/semana & 35 & 21.35 & 11.229 & 859.945 & 5.943 & .005 \\
\hline & Diariamente & 12 & 31.16 & 15.038 & & & \\
\hline \multirow{3}{*}{ DTSintensidad } & Algunas veces/mes & 14 & 14.61 & 10.186 & & & \\
\hline & Varias/semana & 35 & 19.72 & 10.235 & 832.146 & 6.193 & .004 \\
\hline & Diariamente & 12 & 30.50 & 15.934 & & & \\
\hline \multirow{3}{*}{ DTSsumatorio } & Algunas veces/mes & 14 & 30.53 & 20.189 & & & \\
\hline & Varias/semana & 35 & 40.25 & 20.579 & 6423.721 & 6.106 & .026 \\
\hline & Diariamente & 12 & 61.66 & 30.574 & & & \\
\hline
\end{tabular}


Tabla 4

Tabla resumen entre grupos con ANOVA de un factor y prueba post-hoc.

\begin{tabular}{|c|c|c|c|c|c|}
\hline & $\begin{array}{l}\text { Frecuencia } \\
\text { violencia (I) }\end{array}$ & $\begin{array}{l}\text { Frecuencia } \\
\text { violencia }\left(\mathrm{J}_{1}\right)\end{array}$ & $\begin{array}{c}\text { Diferencia de } \\
\text { Medias } \\
\left(\mid-J_{1}\right)\end{array}$ & $\begin{array}{l}\text { Frecuencia de } \\
\text { la violencia }\left(\mathrm{J}_{2}\right)\end{array}$ & $\begin{array}{c}\text { Diferencia de } \\
\text { Medias } \\
\left(1-J_{2}\right)\end{array}$ \\
\hline DTS & Algunas veces/mes & Varias/semana & -6.42 & Diariamente & $-16.23^{* *}$ \\
\hline \multirow[t]{2}{*}{ Frecuencia } & Varias/semana & Algunas veces/mes & $-16.23^{*}$ & Diariamente & -9.81 \\
\hline & Diariamente & Algunas veces/mes & $16.23^{* \star}$ & Varias/semana & 9.81 \\
\hline DTS & Algunas veces/mes & Varias/semana & -5.11 & Diariamente & $-15.88^{* *}$ \\
\hline \multirow[t]{2}{*}{ Intensidad } & Varias/semana & Algunas veces/mes & 5.11 & Diariamente & $-10.77^{\star}$ \\
\hline & Diariamente & Algunas veces/mes & $15.88^{* \star}$ & Varias/semana & $10.77^{\star}$ \\
\hline DTS & Algunas veces/mes & Varias/semana & -9.71 & Diariamente & $-31.12^{* *}$ \\
\hline \multirow[t]{2}{*}{ Total } & Varias/semana & Algunas veces/mes & 9.71 & Diariamente & $-21.40 *$ \\
\hline & Diariamente & Algunas veces/mes & $31.12^{* *}$ & Varias/semana & $21.40^{*}$ \\
\hline
\end{tabular}

\section{Tabla 5}

Diferencias ante el miedo a la separación en cada uno de los indicadores de la escala DTS.

\begin{tabular}{|c|c|c|c|c|c|c|c|c|c|c|}
\hline & \multicolumn{5}{|c|}{ Frecuencia } & \multicolumn{5}{|c|}{ Intensidad } \\
\hline & \multicolumn{2}{|c|}{$\begin{array}{l}\text { No tenían miedo a } \\
\text { la separación }\end{array}$} & \multicolumn{2}{|c|}{$\begin{array}{l}\text { Si tenían miedo a } \\
\text { la separación }\end{array}$} & \multirow[b]{2}{*}{$t$} & \multicolumn{2}{|c|}{$\begin{array}{l}\text { No tenían miedo a } \\
\text { la separación }\end{array}$} & \multicolumn{2}{|c|}{$\begin{array}{l}\text { Sí tenían miedo } \\
\text { a la separación }\end{array}$} & \\
\hline & $M$ & $D T$ & $M$ & $D T$ & & $M$ & $D T$ & $M$ & $D T$ & $t$ \\
\hline 1 & 1.22 & 1.017 & 2.00 & 1.155 & $-2.778^{\star \star}$ & .89 & .747 & 1.68 & 1.030 & $-3.287^{* *}$ \\
\hline 2 & 1.25 & .996 & 2.08 & 1.152 & $-3.001 * \star$ & .97 & .810 & 1.80 & 1.118 & $-3.356^{\star \star \star}$ \\
\hline 3 & .94 & .984 & 1.58 & 1.213 & $-2.244^{\star}$ & .92 & .874 & 1.36 & .995 & -1.840 \\
\hline 4 & 1.08 & .937 & 1.80 & 1.384 & $-2.414^{*}$ & 1.06 & .860 & 1.44 & 1.044 & -1.572 \\
\hline 5 & .97 & .941 & 1.63 & 1.279 & $-2.278^{\star}$ & .89 & .919 & 1.36 & 1.114 & -1.805 \\
\hline 6 & 1.19 & 1.091 & 1.84 & 1.143 & $-2.229^{*}$ & .94 & .791 & 1.60 & 1.000 & $-2.855^{\star \star}$ \\
\hline 7 & 1.17 & .910 & 2.12 & 1.013 & $-3.840 * * *$ & 1.22 & 1.987 & 1.68 & 1.249 & -1.019 \\
\hline 8 & 1.17 & .845 & 1.72 & 1.275 & $-2.040^{\star}$ & .94 & .754 & 1.60 & 1.225 & $-2.587^{\star}$ \\
\hline 9 & 1.06 & .826 & 1.80 & 1.323 & $-2.496^{\star}$ & 1.03 & .845 & 1.84 & 1.214 & $-2.894^{\star *}$ \\
\hline 10 & 1.00 & .793 & 1.56 & 1.121 & $-2.152^{\star}$ & 1.03 & .910 & 1.64 & 1.150 & $-2.222^{\star}$ \\
\hline 11 & .83 & .878 & 1.64 & .860 & $-3.557^{\star \star \star}$ & .97 & 1.014 & 1.64 & .952 & $-2.582^{\star}$ \\
\hline 12 & .86 & 1.018 & 1.52 & 1.085 & $-2.420^{\star}$ & .89 & 1.036 & 1.56 & 1.083 & $-2.443^{\star}$ \\
\hline 13 & .86 & .833 & 1.48 & .918 & $-2.736^{\star \star}$ & .83 & .878 & 1.48 & 1.005 & $-2.665^{\star \star}$ \\
\hline 14 & .83 & .941 & 1.52 & .770 & $-3.012^{\star \star}$ & .72 & .741 & 1.52 & .918 & $-3.747^{* * *}$ \\
\hline 15 & .81 & .856 & 1.84 & .898 & $-4.550 * \star \star *$ & .86 & .899 & 1.56 & .961 & $-2.903^{* \star}$ \\
\hline 16 & .86 & .931 & 1.48 & 1.159 & $-2.309 *$ & .86 & .931 & 1.48 & 1.046 & $-2.428^{\star}$ \\
\hline 17 & .78 & .929 & 1.76 & .970 & $-3.989 * * *$ & .64 & .798 & 1.48 & .963 & $-3.718^{* * *}$ \\
\hline
\end{tabular}


en función de que el maltrato se produjera con distinta frecuencia.

Las diferencias intergrupales a través del estadístico Bonferroni se encuentran principalmente en el grupo de mujeres que padecían maltrato diariamente respecto a los grupos que padecían violencia con menor frecuencia (Tabla 4).

En la Tabla 5 se observan las diferencias entre los dos grupos en función del miedo a la separación en cada uno de los indicadores de la escala DTS. Los resultados evidencian que la percepción de miedo a la separación está relacionada con el estrés postraumático detectado entre las participantes. Los resultados indican que en la mayoría de los reactivos del instrumento se hallan diferencias entre quienes manifestaron tener miedo a la separación y quienes no, tanto en la frecuencia como en la intensidad o afectación del TEPT, especialmente en los síntomas persistentes de aumento de la activación (ítems 13 a 17).

Por otra parte, se encontraron diferencias estadísticamente significativas $(t=-3.740 ; p=$ .000) entre quienes manifestaron miedo a la separación y quienes no respecto al sumatorio de la frecuencia de la escala DTS (Davidson et al., 1997), obteniendo puntuaciones más elevadas entre quienes afirmaron tener miedo a la separación $(M=28.60 ; D T=12.612)$ que quienes no $(M=16.88 ; D T=11.155)$. En cuanto a la intensidad o afectación, también se encontraron diferencias estadísticamente significativas $(t=-3.573 ; p=.000)$ entre

Tabla 6

Pesos de regresión estandarizada de las relaciones causales del modelo.

\begin{tabular}{|c|c|c|c|c|}
\hline \multicolumn{2}{|c|}{ Relaciones causales } & $\begin{array}{c}\text { Pesos de regresión } \\
\text { estandarizada }\end{array}$ & P \\
\hline $\begin{array}{c}\text { Frecuencia del maltrato } \\
\text { sufrido }\end{array}$ & $\rightarrow$ & $\begin{array}{c}\text { Frecuencia del estrés } \\
\text { postraumático }\end{array}$ & .449 & .000 \\
\hline $\begin{array}{c}\text { Frecuencia del estrés } \\
\text { postraumático }\end{array}$ & $\rightarrow$ & $\begin{array}{c}\text { Intensidad del estrés } \\
\text { postraumático }\end{array}$ & .945 & .000 \\
\hline $\begin{array}{c}\text { Intensidad del estrés } \\
\text { postraumático }\end{array}$ & $\rightarrow$ & Miedo a la separación & .435 & .000 \\
\hline $\begin{array}{c}\text { Intensidad del estrés } \\
\text { postraumático }\end{array}$ & $\rightarrow$ & Ansiedad y depresión & .652 & .000 \\
\hline
\end{tabular}

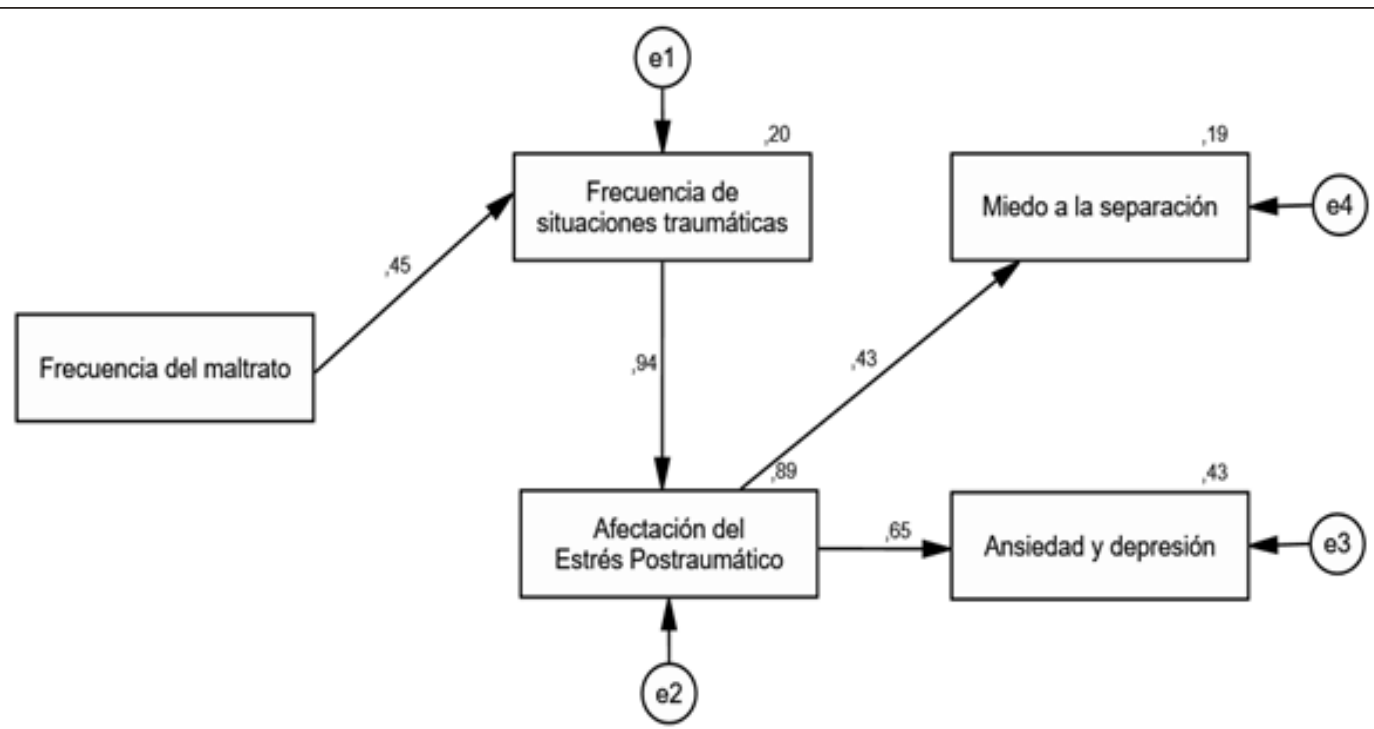

Figura 1: Modelo estructural propuesto. 
ambos grupos $(M=26.72 ; D T=12.039)$ y $(M=16.02 ; D T=10.974)$. Finalmente, se hallaron diferencias $(t=-3.532 ; p=.001)$ en el sumatorio total de la escala respecto quienes manifestaron tener miedo a la separación ( $M$ $=54.82 ; D T=24.231)$ y quienes no $(M=$ 33.34; DT $=21.584)$. Esta circunstancia no parece estar relacionada con la escala K-10 (Kessler et al., 1992) ( $t=-1.528 ; p=.132)$ $(M=28.11$; DT $=4.670)$ vs. $(M=30.72 ; D T$ $=8.60)$.

Antes de realizar el modelo de ecuaciones estructurales, se calculó la normalidad multivariada. Los resultados reportaron un coeficiente Mardia de 6.04, por lo que se optó por el método de estimación de Máxima Verosimilitud. El modelo analiza las relaciones directas e indirectas entre cinco variables observables (Figura 1): la frecuencia de la violencia sufrida, la frecuencia de las situaciones traumáticas, la intensidad o afectación por estrés postraumático, el miedo al abandono y la puntuación en la escala de ansiedad y depresión. La calidad de ajuste del modelo es adecuada y todas las pruebas están en el rango de aceptabilidad: $\chi^{2}=4.97 ; g l=6$; valor de $p$ $=.548 ; \chi^{2} / \mathrm{gl}=.83 ; \mathrm{GFI}=.97 ; \mathrm{AGFI}=.92 ;$ $\mathrm{NFI}=.98 ; \mathrm{CFI}=1.00 ; \mathrm{RMSEA}=.00 ; \mathrm{RMSR}$ $=.04$. Tal y como se puede ver, el modelo estructural explica el $20 \%$ de la frecuencia del TEPT, el $89 \%$ de la intensidad o afectación del TEPT, el $19 \%$ del miedo a la separación y el 43\% de la ansiedad y depresión.

Para analizar la adecuación del modelo propuesto, se estudió la significación de las relaciones entre las distintas variables (Tabla 6). Todos los pesos de regresión son significativos al nivel de $p<.05$. La afectación por TEPT tiene un efecto directo en el miedo a la separación ( $\beta$ $=.435 ; \mathrm{p}=.000$ ) y en los niveles de ansiedad y depresión $(\beta=.652 ; p=.000)$. Asimismo, la frecuencia del maltrato sufrido tiene un impacto indirecto en la intensidad o afectación por TEPT y un impacto directo en la frecuencia de dicho trastorno $(\beta=.449 ; p=.000)$.

\section{DISCUSIÓN Y CONCLUSIONES}

En el presente estudio se analiza la relación entre la salud mental de mujeres víctimas de violencia de pareja y el miedo a la separación, específicamente, la frecuencia e intensidad del TEPT y su influencia en el miedo a la ruptura con el agresor. Para empezar, la totalidad de las mujeres que formaron parte de la investigación padecieron violencia psicológica y física. Más del $70 \%$ fueron forzadas a mantener relaciones sexuales con la pareja. Hay que añadir que las distintas agresiones se producían con bastante frecuencia (varias veces a la semana) en más de la mitad de los casos y una de cada cinco lo padecía diariamente. Estos datos revelan la problemática de un país sobre el que no se han reportado suficientes datos (Ellsberg y Emmelin, 2014). Además, este tipo de situaciones traumáticas podrían haber alterado la salud mental de las mujeres víctimas (Echeburúa et al., 2016), paralizando los procesos de separación del agresor debido al deterioro de su bienestar y calidad de vida (Ruiz et al., 2013; Sarasua et al., 2007).

En cuanto al estado de salud psicológica, la puntuación media de la muestra indica una alta probabilidad de padecer TEPT, por encima del punto de corte. En cada uno de los reactivos para medir la frecuencia del TEPT de la escala de Davidson et al. (1997) se hallaron puntuaciones en torno al valor "una vez a la semana", que indica baja frecuencia respecto a los recuerdos angustiosos, la evitación de estímulos asociados al trauma y las reacciones fisiológicas derivadas de un acontecimiento traumático. En lo que respecta a la intensidad o afectación de tales indicadores, la puntuación es ligeramente inferior, en torno al valor "me ha afectado poco". Los datos parecen indicar que, a pesar de la situación de violencia, solo el $8.1 \%$ de las entrevistadas puntuaron por encima del valor 3 tanto en frecuencia como en intensidad o afectación a los reactivos del DTS (Davidson et al., 1997). En síntesis, en la muestra de mujeres nicaragüenses, el TEPT tiene una menor prevalencia respecto a otros estudios (Golding, 1999; Greene et al., 2018; Mbalo et al., 2017; Mertin y Mohr, 2000; Riggs et al., 2000). Estos datos podrían estar afectados por posibles sesgos en las respuestas, pues la evaluación del TEPT es compleja dada la alta comorbilidad hallada en otras investigaciones (Arce et al., 2015). 
No obstante, en cuanto a la frecuencia con la que la violencia tenía lugar y la relación con la salud psicológica de las participantes, se han encontrado diferencias entre quienes padecía maltrato diariamente respecto a quienes lo sufrían en menor medida. En este sentido, los resultados reportan que los episodios de violencia padecidos diariamente están relacionados con una mayor puntuación en la escala DTS (Davidson et al., 1997). Además, más de la mitad de las entrevistadas alcanzaron puntuaciones elevadas en ansiedad y depresión, y una de cada tres participantes alcanzó puntuaciones muy altas en la escala K-10 (Kessler et al., 1992). Por lo tanto, la violencia que han padecido por parte de la pareja parece afectar a la salud psicológica y al bienestar de estas mujeres en línea con otras investigaciones (Mbalo et al., 2017; Sullivab y Weiss, 2017 ).

Por otra parte, como se ha reportado en estudios previos en otros países, que las mujeres no rompan con la situación de maltrato podría estar condicionado por muchos factores (Bosch et al., 2005; Ruiz et al., 2013; Watts y Zimmerman, 2002). En particular, el miedo a la separación parece afectar a un alto porcentaje de la muestra, ya que el $59 \%$ de las participantes afirmaron tenerlo. Además, aunque el TEPT no ha afectado a la mayoría de las participantes, se han encontrado diferencias estadísticamente significativas en cada uno de los indicadores del DTS al comparar a quienes manifestaron miedo a la separación y quienes no percibían ese temor. Las mayores diferencias se encontraron respecto a la frecuencia e intensidad o afectación en "pesadillas con el acontecimiento", "haberse sentido irritable - haber tenido episodios de ira o rabia intensa" y "haberse sobresaltado o asustado fácilmente". Por lo tanto, la sintomatología del TEPT parece estar asociada al miedo a la separación, que podría estar inhibiendo la ruptura con la relación de abuso (Bolaños et al., 2009; Ruiz et al., 2013; Sullivan et al., 2018). De acuerdo con algunos autores, la separación del agresor representa un periodo crítico en el que podría haberse incrementado e intensificado la violencia dada la frecuencia del maltrato que padecían las participantes, situación que paralizaría la decisión de iniciar una vida sin violencia (Goodman et al., 1999; Heise et al., 1999; Ruiz et al., 2013; Sagot y Carcedo, 2000). En el presente estudio se encontraron diferencias respecto al miedo a la separación en el sumatorio total de la escala DTS, así como respecto a la frecuencia y a la intensidad en dicho instrumento. Padecer TEPT y otros problemas de salud grave podrían estar impidiendo a las mujeres el uso de recursos personales para romper con la situación de maltrato (Khalifeh et al. , 2015; Luna et al., 2018; Sparrow et al., 2017). No obstante, a pesar de la prevalencia en la muestra de trastornos de ansiedad y depresión, estos no están relacionadas con el miedo a la separación. Por lo tanto, el TEPT y otros trastornos psicológicos requieren de una cuidadosa evaluación, puesto que se han encontrado en otros estudios falsos positivos y negativos ante las limitaciones de los instrumentos y su aplicación tradicionalmente clínica que no se adapta a todos los contextos (Arce et al., 2015; Fariña et al., 2014).

Tal y como indica el modelo de ecuaciones estructurales, una mayor frecuencia de la violencia aumenta la frecuencia del TEPT, que está relacionada con una mayor intensidad o afectación del trastorno, el cual incrementa el deterioro en la salud psicológica en cuanto al padecimiento de ansiedad y depresión. Sin embargo, estos problemas de salud no se relacionan con el miedo a la separación, siendo la intensidad de la sintomatología del TEPT la que afecta a la percepción de ese miedo. Los resultados parecen indicar que, aun no cumpliendo con la totalidad de los indicadores, este trastorno puede alterar la percepción de control en uno de los momentos más difíciles por los que atraviesan las mujeres víctimas de violencia de pareja en lo que respecta a la ruptura con la relación de maltrato (Anderson y Saunders, 2003; Heise et al., 1999; Lerner y Kennedy, 2000; Ruiz et al., 2013; Sagot y Carcedo, 2000; Sarasúa et al., 2007).

En el presente estudio cabe señalar una serie de limitaciones. Para empezar, la muestra está formada por un número reducido de participantes que hace difícil poder generalizar los resultados encontrados. Por otra parte, el miedo a la separación se ha evaluado mediante 
una pregunta ad hoc con respuesta dicotómica y no mediante un instrumento validado, lo que puede introducir un sesgo en su medición. Además, del miedo a la separación pueden desprenderse variables de origen muy diverso que podrían estar generando ese temor, como el miedo a las consecuencias, a no saber dónde ir, miedo a la soledad, miedo a la desintegración familiar o algún tipo de dependencia hacia el agresor (Bosch et al., 2005; Watts y Zimmerman, 2002). En este sentido, podrían desarrollarse instrumentos con los que se evalúe la intensidad de dicho miedo y se analice su origen, dado que este puede ser multicausal. Asimismo, tanto en el miedo como en los niveles de TEPT y otros problemas de salud pueden estar mediando otras variables relacionadas con la personalidad, como la capacidad de resiliencia y las fuentes de apoyo social que serían factores de protección y potenciadores de bienestar. Por ello, desde una perspectiva aplicada, es fundamental contar con una amplia variedad de instrumentos de evaluación, como entrevistas estructuradas, modelos narrativos y pruebas psicofisiológicas que ofrezcan buenas propiedades psicométricas y que resulte sencilla su aplicación en función de que el objetivo sea clínico, forense o de prevalencia (Echeburua et al., 2016).

Como futuras líneas de investigación sería interesante analizar estas variables desde diseños longitudinales, ya que permitiría obtener resultados más concluyentes. De la misma manera, analizar la relación entre el TEPT y otros problemas de salud en mujeres que se encuentran en distintos momentos del proceso de ruptura esclarecería y permitiría generar indicadores que facilitasen y reforzasen el apoyo y el seguimiento desde distintos perfiles de profesionales, teniendo en cuenta que el enfoque de la intervención con víctima de violencia de pareja habría de ser interprofesional y desde perspectivas multidisciplinares.

Aun con todo, se muestran datos de un país sobre el que no se han realizado suficientes estudios de prevalencia $y$, en menor medida, respecto a la salud mental de mujeres víctimas de violencia de pareja (Ellsberg y Emmelin, 2014) y cómo influye en el mantenimiento de la convivencia con el agresor. En Nicaragua, la vulneración de los derechos de las mujeres se incrementa cuando no hay dispositivos de ayuda que faciliten la intervención o estos son muy limitados, por lo que la ausencia de recursos y la falta de apoyo hacen que las mujeres tengan dificultades para salir de la situación de maltrato. Esto es especialmente grave atendiendo a la alta prevalencia de la violencia contra las mujeres en la región (Boyce et al., 2016). Además, las circunstancias de precariedad del país (PNUD, 2016) impiden que se implementen medidas de atención e intervención por parte de profesionales (Colombini et al., 2008). Por ello es importante proporcionar recursos adecuados al contexto nicaragüense, tanto para visibilizar el problema, como para eliminar la violencia contra las mujeres (Salazar et al., 2009). Disponer de organismos que desarrollen apoyo integral a las mujeres es una tarea prioritaria por parte de los diferentes Estados para poner fin a este problema de salud pública y atentado contra los derechos humanos (Boyce et al., 2016; Silva y Azeredo, 2019; Rivas et al., 2018).

- Conflicto de intereses.

Los autores declaran no tener ningún conflicto de intereses.

\section{REFERENCIAS}

American Psychiatric Association. (2014). Manual diagnóstico y estadístico de los trastornos mentales (5a ed.). Madrid, España: Panamericana.

Anderson, D. K., y Saunders, D. G. (2003). Leaving an abusive partner: An empirical review of predictors, the process of leaving and psychological well-being. Trauma, Violence and Abuse, 4, 163-191. http://doi. org/10.1177/1524838002250769

Arce, R., Fariña, F., y Vilariño, M. (2015). Daño psicológico en casos de víctimas de violencia de género: Estudio comparativo de las evaluaciones forenses. Revista Iberoamericana de Psicología y Salud, 6(2), 72-80. http://doi. org/10.1016/i.rips.2015.04.002

Bolaños, M. L., Gutiérrez Rodríguez, L., Pérez, E. M., Fanjul, C., Pérez de Matus, M. A., y Barres, O. (2009). Diagnóstico de violencia intrafamiliar y sexual en Nicaragua. Nicaragua: Programa de las Naciones Unidas para el Desarrollo (PNUD). 
Bosch, E., Ferrer, V., Alzamora, A., y Navarro, C. (2005). Itinerarios hacia la libertad: La recuperación integral de las víctimas de violencia de género. Psicología y Salud, 15(1), 97-105.

Bosch, E., Ferrer, V., Ferreiro, V., y Navarro, C. (2013). La violencia contra las mujeres: El amor como coartada. Barcelona, España: Anthropos. Boyce, S., Zeledón, P., Téllez, E., y Barrington, C. (2016). Gender-Specific jealousy and infidelity norms as sources of sexual health risk and violence among Young coupled Nicaraguans. American Journal of Public Heath, 106(4), 625-632. https://doi.org/10.2105/ AJPH.2015.303016

Colombini, M., Mayhew, S., y Watts, C. (2008). Health-sector responses to intimate partner violence in low- and middle-income setting: A review of current models, challenges and opportunities. Bulletin of the World Health Organization, 86(8), 635-642. https://doi. org/10.2471/BLT.07.045906

Davidson, J. R. T., Book, S. W., Colket, J. T., Tupler, L. A., Roth, S., David, D., ... y Davidson, R. M. (1997). Assesment of a new self-rating scale post-traumatic stress disorder. Psychological Medicine, 27(1), 153-160. http://doi. org/10.1017/S0033291796004229

Echeburúa, E., Amor, P. J., Sarasua, B., Zubizarreta, I., Holgado-Tello, F. P., y Muñoz, J. M. (2016). Escala de Gravedad de Síntomas Revisada (EGS-R) del trastorno de Estrés Postraumático según el DSM-5: Propiedades psicométricas. Terapia Psicológica, 34(2), 111 -128.

Ellsberg, M. y Emmelin, M. (2014). Intimate partner violence and Mental Health. Global Health Action, 7. https://doi.org/10.3204/ gha.v7.25658

Fariña, F., Arce, R., Vilariño, M., y Novo, M. (2014). Assessment of the standard forensic procedure for the evaluation of psychological injury in intimate-partner violence. Spanish Journal of Psychology, 17(e32), 1-10. http:// doi.org/10.1017/sip.2014.30

Golding, J. M. (1999). Intimate partner violence as a risk factor for mental disorders: A metaanalysis. Journal of Family Violence, 14, 99-132. http://doi.org/10.1023/A:1022079418229
Goodman, L. A., Bennet, L., y Dutton, M. A. (1999). Obstacles to victim's cooperation with the criminal prosecution of their abusers: The role of social supports. Violence and Victims, 21 (5), 661-624. http://doi.org/10.1891/08866708.14 .4 .427

Greene, C., Chan, G., McCarthy, K., Wakschlag, L., y Briggs-Gowan, M. (2018). Psychological and physical intimate partner violence and Young children's mental health: The role of maternal posttraumatic stress symptoms and parenting behaviors. Child Abuse \& Neglect, 77, 168-179. https://doi.org/10.1016/i. chiabu.2018.01.012

Guerra, C., Martínez, P., Ahumada, C., y Díaz, M. (2013). Análisis psicométrico preliminar de la Escala de Trauma de Davidson (DTS) en adolescentes chilenos. Suma Psicológica, 10(2), $41-48$.

Heise, L., Ellsberg, M., y Gottemoeller, M. (1999). Ending violence against women. Population Reports, 27(4), 1 - 1.

Kessler, R., Andrews, G., Cople, L. J., Hiripi, E., Mroczek, D. K., Normand, S. L., ..., y Zaslaysky, A. M. (2002). Short screening scales to monitor population prevalence and trends in nonspecific psychological distress. Psychological Medicine, 32(6), 959-976. https://doi. $\mathrm{org} / 10.1017 / \mathrm{S} 0033291702006074$

Khalifeh, H., Oram, S., Trevillion, K., Johnson, S., y Howard, L. M. (2015). Recent intimate partner violence among people with chronic mental illness: Findings from a national crosssectional survey. British Journal of Psychiatry, 207(3), 207-212. https://doi.org/10.1192/ bip.bp. 114.144899

Lerner, C. F., y Kennedy, C. T. (2000). Stay-Leave Decision making in Battered Women: Trauma, Coping and Self_Efficacy. Cognitiva Therapy and Research, 24(2), 215-232. https://doi. org/10.1023/A: 1005450226110

Luna, J. A., Rodríguez, P. D., y Hernández, I. (2018). Perfil neuropsicológico de atención y en víctimas del conflicto armado colombiano. Revista de Psicología, 36(2), 701 -718. https:// doi.org/10.18800/psico.201802.011

Mardia, K. V. (1970). Measures of multivariate skewness and kurtosis with applications. 
Biometrika, 57(3), 519.530. https://doi. org/10.1093/biomet/57.3.519

Mbalo, N., Zhang, M., y Sam, N. (2017). Risk factors for PTSD and Depression in female survivors of rape. Psychological Trauma: Theory, Research, Practice, and policy, 9(3), 301-308. https://doi.org/10.1037/tra0000228

Mertin, P. y Mohr, P. (2000). Incidence and correlates of posttraumatic stress disorder in Australian victims of domestic violence. Journal of Family Violence, 15(4), 411 -422. https:// doi.org/10.1023/A:1007510414571

Programa de las Naciones Unidas para el Desarrollo (2016). Human Development Report 2016. New York: PNUD.

Riggs, D. S., Caulfield, M. B., y Street, A. E. (2000). Risk for domestic violence: Factors asociated with perpetration and victimizacion. Journal of Clinical Psychology, 56(10), 1289-1316. http://doi.org/10.1002/10974679(200010)56:10<1289::AIDJCLP4 > 3.0.CO;2-Z

Rivas, E., Panadero, S., Bonilla, E., Vázquez, R., y Vázquez, J. J. (2018). Influencia del apoyo social en el mantenimiento de la convivencia con el agresor en víctimas de violencia de género de León (Nicaragua). Informes Psicológicos, 18(1), 145-165. http://doi.org/10.18566/infpsic. v18n1a08

Rivas, E., Bonilla, E., y Vázquez, J.J. (2019). Influence of the history of abuse and suicidal attempts behavior among women victims of violence in Nicaragua. Journal of Community of Psychology, 1-71. http://doi.org/10.1002/ jcop. 22260

Ruiz-Hernández, M., López-Angulo, L., HernándezCabrera, Y., Castañeda-Álvarez, E., y ÁguilaRodríguez, Y. (2013). Caracterización de las mujeres maltratadas por su pareja desde la perspectiva de género. Medisur, 17 (1), 15-26.

Sagot, M., y Carcedo, A. (2000). La Ruta Crítica de las mujeres afectadas por la violencia intrafamiliar en América Latina. Pan American Health Organization.

Salazar, M., Valladares, E., Óhman, A., y Högberg, U. (2009). Ending Intimate Partner Violence after pregnancy: Findings from a community-based longitudinal study in Nicaragua. BMC Public
Health, 9, 350. http://doi.org/10.1086/14712458-9-350

Sarasúa, B., Zubizarreta, I., Echeburúa, E., y Corral, P. (2007). Perfil psicopatológico diferencial de las víctimas de violencia de pareja en función de la edad. Psicothema, 19(3), 459-466.

Schreiber, J. B., Nora, A., Stage, F. K., Barlow, E. A., y King, J. (2006). Reporting Structural Equation Modeling and Confirmatory Factor Analysis Results: A Review. The Journal of Educational Research, 99(6), 323-337. https:// doi.org/10.3200/JOER.99.6.323-338

Silva, A. N., y Azeredo, C. (2019). The association between victimization resulting from intimate partnerviolence and depression among Brazilian adults. Ciencia y Salude Colectiva, 24(7), 2691-2700. https://doi.org/10.1590/141381232018247.25002017

Sparrow, K., Kwan, J., Howard, L., y MacManus, D. (2017). Systematic review of mental health disorders and intimate partner violence victimization among military populations. Social Psychiatry and Psychiatric Epidemiology, 52(9), 1059-1080. http://doi.org/10.1007/s00127. 017-1423-8

Sullivan, T., y Weiss, N. H. (2017). Is firearm threat in intimate relationships associated with posttraumatic stress disorder symptoms among women? Gender and Violence, 4(2), 31-36. http://doi.org/10.1089/vio.2016.0024

Sullivan, T., Weiss, N. H., Price, C., Pugh, N., y Hansen, N. B. (2018). Strategies for coping with individual PTSD symptoms: Experiences of African American victims of intimate partner violence. Psychological Trauma: Theory, Research, Practice y Policy, 10(3), 336-344. http://doi.org/10.1037/tra0000234

Vargas, B. E., Villamil, V., Rodríguez, C., Pérez, J., y Cortés, J. (2011). Validación de la escala Kessler 10 (K-10) en la detección de depresión y ansiedad en el primer nivel de atención: Propiedades psicométricas. Salud Mental, 34(4), 323-331.

Watts, C., y Zimmerman, C. (2002). Violence against women: global scope magnitude. The Lancet, 359, 1232-1237. https://doi. org/10.1016/50140-6736(02)08221-1 